\title{
Performance bound for quantum absorption refrigerators
}

\author{
Luis A. Correa, ${ }^{1,2,3,}{ }^{*}$ José P. Palao, ${ }^{2,4}$ Gerardo Adesso, ${ }^{1}$ and Daniel Alonso ${ }^{2,3}$ \\ ${ }^{1}$ School of Mathematical Sciences, The University of Nottingham, University Park, Nottingham NG7 2RD, United Kingdom \\ ${ }^{2}$ IUdEA Instituto Universitario de Estudios Avanzados, Universidad de La Laguna, La Laguna 38203, Spain \\ ${ }^{3}$ Departamento Física Fundamental, Experimental, Electrónica y Sistemas, Universidad de La Laguna, La Laguna 38203, Spain \\ ${ }^{4}$ Departamento de Física Fundamental II, Universidad de La Laguna, La Laguna 38204, Spain \\ (Received 19 December 2012; revised manuscript received 4 April 2013; published 29 April 2013)
}

\begin{abstract}
An implementation of quantum absorption chillers with three qubits has been recently proposed that is ideally able to reach the Carnot performance regime. Here we study the working efficiency of such self-contained refrigerators, adopting a consistent treatment of dissipation effects. We demonstrate that the coefficient of performance at maximum cooling power is upper bounded by $3 / 4$ of the Carnot performance. The result is independent of the details of the system and the equilibrium temperatures of the external baths. We provide design prescriptions that saturate the bound in the limit of a large difference between the operating temperatures. Our study suggests that delocalized dissipation, which must be taken into account for a proper modeling of the machine-baths interaction, is a fundamental source of irreversibility which prevents the refrigerator from approaching the Carnot performance arbitrarily closely in practice. The potential role of quantum correlations in the operation of these machines is also investigated.
\end{abstract}

DOI: 10.1103/PhysRevE.87.042131

\section{INTRODUCTION}

The study of quantum thermal machines has attracted increasing attention over recent years. This is motivated on one hand by the fundamental interest in understanding the emergence of basic thermodynamic principles at the quantum mechanical level [1-12], and on the other hand, by the potential technological applications of these machines, for instance to control the heat transport in nanoengineered devices [13-16]. In particular, several models have been proposed [1-7] realizing quantum absorption chillers, that is, refrigerators in which the external source of work is replaced by a heat bath. A realization of such refrigerators, which has been introduced in [5-7], consists of three interacting qubits, with a vanishingly small interaction strength, each one in contact with a heat bath. In spite of the technological challenges behind its physical implementation, this machine can be experimentally realized, e.g., with superconducting qubits or arrays of quantum dots $[14,15]$. Furthermore, its operation may be understood in a very neat way, providing a physical insight into the sources of irreversibility in absorption chillers [7].

It has been predicted that, with a suitable choice of the machine parameters, such a refrigerator can ideally attain a coefficient of performance (COP) reaching the Carnot bound $\varepsilon_{C}$ [6]. However, we argue that the central assumption of vanishing mutual interaction between the refrigerator qubits cannot be realistically maintained. As long as the interaction is finite, each bath will exchange energy with the whole three-qubit system, rather than just locally with the single qubit to which it is connected. This is usually the case with any interacting multiparticle dissipative system. As we shall show, the resulting delocalized dissipation prevents the refrigerator from approaching the Carnot limit arbitrarily closely, thus embodying a fundamental source of irreversibility that is expected to arise in all concrete implementations.

*1acorrea@ull.es
This situation is reminiscent of that of realistic heat engines or cooling cycles, topical areas of study in finitetime thermodynamics. There, the finite heat transfer rates constitute the essential source of irreversibility which makes the Carnot bound unattainable in practice. For this reason, an important line of research deals with devising alternative, tight performance bounds, such that some suitable figure of merit of the machine under consideration is maximized [17-21]. In this spirit, we address the following question: What is the highest achievable COP at maximum cooling power for the quantum absorption refrigerators of Refs. [5-7]? Answering this question would provide a practical performance bound against which the efficiency of any future realization of these machines could be benchmarked.

Another relevant and related question to ask is whether the "quantumness" of the refrigerator, as revealed for instance by the stationary quantum discord [22,23], plays any role in its operation. This would help to unveil connections between quantum correlations and efficient energy transport out of equilibrium that so far have remained elusive.

In this paper we answer both questions. In the first place, by considering unstructured bosonic baths and a consistent dissipative qubit-bath interaction, we find that the COP at maximum power is tightly upper bounded by $\frac{3}{4} \varepsilon_{C}$, where $\varepsilon_{C}=\left(1-\frac{T_{h}}{T_{w}}\right) /\left(\frac{T_{h}}{T_{c}}-1\right)$ is the Carnot COP and $\left\{T_{w}, T_{h}, T_{w}\right\}$ are the three temperatures between which the refrigerator operates. We also give sufficient conditions to saturate this bound in the limit of large temperature difference $T_{c} / T_{h} \ll 1$. Second, we issue a comprehensive analysis of stationary bipartite quantum correlations in the various relevant qubit-qubit partitions. Although a nonvanishing discord is always found in a specific partition, it does not relate to the stationary heat flows, reinforcing the idea that this family of thermal machines operates in an effectively classical way [7], despite having a genuinely quantum physical support.

The paper is organized as follows: In Sec. II we introduce the microscopic model of the three-qubit refrigerator object of our study. In Sec. III we address its reduced dynamics via a 
Lindblad-type master equation (whose derivation is deferred to Appendix A) that allows for a consistent treatment of dissipation. We also point out how the delocalized dissipation effects, unavoidable in practice, prevent the refrigerator from being maximally efficient. In Sec. IV we demonstrate the existence of general upper bounds on the coefficient of performance of the refrigerator at maximum cooling power, and provide design prescriptions to saturate such bounds (a supporting analytical proof is presented in Appendix B). In Sec. V we report our complete study of stationary bipartite quantum correlations in the system. Finally, we draw our conclusions in Sec. VI.

\section{PRELIMINARIES}

Let us begin by introducing the total Hamiltonian of the refrigerator. The Hilbert space of the system is $\mathcal{H}_{S}=\mathcal{H}_{S, w} \otimes$ $\mathcal{H}_{S, h} \otimes \mathcal{H}_{S, c}$, where we label the three qubits as "work," "hot," and "cold" ( $w, h$, and $c)$, after the heat baths to which each of them is connected (see Fig. 1). Their free Hamiltonians are

$$
H_{0, \alpha}=\omega_{\alpha}\left|1_{\alpha}\right\rangle\left\langle 1_{\alpha}\right|,
$$

where $\alpha=\{w, h, c\}$, and we work in natural units $\hbar=k_{B}=1$. The corresponding bosonic baths are given by

$$
H_{B, \alpha}=\sum_{\lambda} \omega_{\lambda} a_{\alpha, \lambda}^{\dagger} a_{\alpha, \lambda} .
$$

As local qubit-bath dissipative coupling, we choose terms of the form

$$
H_{D, \alpha}=\sqrt{\gamma}\left(c_{x_{\alpha}} \sigma_{x_{\alpha}}+c_{y_{\alpha}} \sigma_{y_{\alpha}}\right) \otimes \sum_{\lambda} g_{\lambda}\left(a_{\alpha, \lambda}-a_{\alpha, \lambda}^{\dagger}\right),
$$

where $\left\{c_{x_{\alpha}}, c_{y_{\alpha}}\right\} \in \mathbb{R}$, and $g_{\lambda} \propto \sqrt{\omega_{\lambda}}$ to ensure flat spectral densities $J(\omega) \sim g_{\alpha, \lambda}^{2} / \omega_{\alpha, \lambda}$ [24]. Here, we absorbed the order of magnitude of $J(\omega)$ into the dissipation rate $\gamma$. With no loss of generality, we can set $c_{x_{\alpha}}=1$ and $c_{y_{\alpha}}=0$. This kind of system-environment coupling stands, e.g., for the dipole interaction between a two-level atom and the electromagnetic field at thermal equilibrium [24].

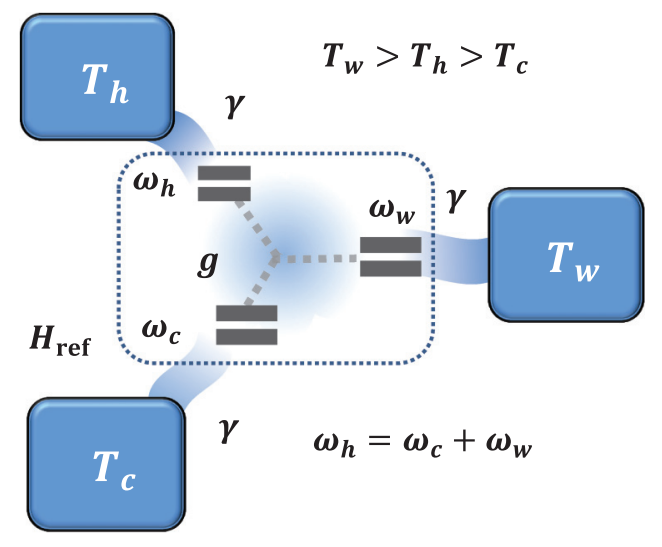

FIG. 1. (Color online) Schematic representation of the three-qubit absorption refrigerator. The refrigerator qubits dissipate into their respective baths, with equilibrium temperatures $T_{w}>T_{h}>T_{c}$, at a rate $\gamma$. The three-body interaction, of strength $g$, allows for energy exchange between the refrigerator qubits, whose energy spacings $\omega_{\alpha}$ are required to satisfy $\omega_{h}=\omega_{c}+\omega_{w}$.
It only remains to specify the three-body interaction between the qubits, which in our case is

$$
H_{I}=g\left(\left|1_{w} 0_{h} 1_{c}\right\rangle\left\langle 0_{w} 1_{h} 0_{c}\right|+\text { H.c. }\right),
$$

where $g$ is the interaction strength. The qubit energies are chosen as $\omega_{h}=\omega_{c}+\omega_{w}\left(\omega_{h}>\omega_{w}\right)$, so that the subspace $\left\{\left|1_{w} 0_{h} 1_{c}\right\rangle,\left|0_{w} 1_{h} 0_{c}\right\rangle\right\}$ is approximately degenerate, as long as $g \ll 1$.

The total Hamiltonian is then finally

$$
H_{T}=\sum_{\alpha} H_{0, \alpha}+H_{I}+\sum_{\alpha} H_{D, \alpha}+\sum_{\alpha} H_{B, \alpha} .
$$

We now briefly explain how the operation of the refrigerator may be understood (see [7] for details). In the ideal scenario of vanishing $g$, the reduced stationary state of the work and hot qubits, $\varrho_{w, h}^{\infty} \equiv \operatorname{Tr}_{c} \varrho$, has high fidelity with $\tau_{w} \otimes \tau_{h}$, where $\tau_{\alpha}=Z_{\alpha}^{-1} e^{-H_{0, \alpha} / T_{\alpha}}$ stands for the thermal state of qubit $\alpha$ at the equilibrium temperature $T_{\alpha}$, and $\varrho$ denotes the reduced state of the three qubits after tracing out the heat baths.

It follows that the truncation of $\varrho_{w, h}^{\infty}$ into the twodimensional subspace $\mathcal{H}_{S, v}$ of $\mathcal{H}_{S, w} \otimes \mathcal{H}_{S, h}$ spanned by $\left\{\left|1_{w} 0_{h}\right\rangle,\left|0_{w} 1_{h}\right\rangle\right\}$, which defines a "machine virtual qubit" $v$, has an effective virtual temperature approximately given by

$$
T_{v} \equiv \frac{\omega_{h}-\omega_{w}}{\omega_{h} / T_{h}-\omega_{w} / T_{w}}
$$

The interaction $H_{I}$ allows the cold qubit to exchange energy with the machine virtual qubit, while being simultaneously thermalized by the cold bath through $H_{D, c}$. Suitable choice of frequencies and temperatures may result in $T_{v}<T_{c}$, so that the (nonequilibrium) stationary state $\varrho_{c}^{\infty}$ is effectively colder than $T_{c}$. The excited state population deficit in $\varrho_{c}^{\infty}$ is compensated by a net energy transfer from the cold bath (which stands for the object to cool) into the refrigerator. This is what we shall understand by cooling. The machine therefore just mediates between the cold object at temperature $T_{c}$ and a suitably filtered virtual temperature $T_{v}$. Thermalization is then responsible for cooling within the cooling window $0 \leqslant T_{v} \leqslant T_{c}$, or in terms of the cold frequency $\omega_{c}$

$$
0<\omega_{c}<\frac{\left(T_{w}-T_{h}\right) T_{c}}{\left(T_{w}-T_{c}\right) T_{h}} \omega_{h} .
$$

When $T_{v}=T_{c}$ and always under the assumption of localized dissipation, which is consistently realized only for vanishing $g$, the refrigerator would in principle saturate the Carnot bound $\varepsilon_{C}$ on the COP [6].

\section{REALISTIC MODELING OF THE DISSIPATION}

\section{A. The quantum master equation}

We shall now extend the model of Refs. [5-7] to consistently account for the dissipative dynamics of the refrigerator in the realistic scenario of even very small but nonvanishing coupling strength $g$. We can derive a general equation of motion for the qubits from first principles, by employing the standard Born-Markov assumption of weak-memoryless system-environment interaction [24]. Such master equation, whose complete derivation is reported in Appendix A, is 
written as

$$
\begin{aligned}
\dot{\varrho} & =-i\left[H_{\mathrm{ref}}, \varrho\right]+\sum_{\alpha} \mathcal{D}_{\alpha}[\varrho] \\
& =-i\left[H_{\mathrm{ref}}, \varrho\right]+\sum_{\alpha, \omega} \Gamma_{\alpha, \omega}\left(A_{\alpha, \omega} \varrho A_{\alpha, \omega}^{\dagger}-\frac{1}{2}\left\{A_{\alpha, \omega}^{\dagger} A_{\alpha, \omega}, \varrho\right\}_{+}\right),
\end{aligned}
$$

where $H_{\text {ref }} \equiv \sum_{\alpha} H_{0, \alpha}+H_{I}$. The spectral correlation tensor, denoted by $\Gamma_{\alpha, \omega}$, is proportional to the real part of the power spectra of the bath correlation functions. Note that since the heat baths are independent, the correlation tensor is diagonal in $\alpha$. We use its explicit form for electromagnetic radiation at thermal equilibrium: $\Gamma_{\alpha, \omega} \propto \omega^{3} \exp \left(\omega / 2 T_{\alpha}\right)\left(\sinh \omega / 2 T_{\alpha}\right)^{-1}$ [24].

The non-Hermitian Lindblad operator $A_{\alpha, \omega}$ associated with the bath $\alpha$ performs transitions of frequency $\omega$ at rate $\Gamma_{\alpha, \omega}$, between the ( $g$-independent) eigenstates of the refrigerator Hamiltonian $H_{\text {ref }}$. They result from the decomposition of the system-environment couplings $\left(\sqrt{\gamma} \sigma_{x_{\alpha}}=\sum_{\omega} A_{\alpha, \omega}\right)$ as eigenoperators of the refrigerator Hamiltonian ( $\left[H_{\mathrm{ref}}, A_{\alpha, \omega}\right]=$ $\left.-\omega A_{\alpha, \omega}\right)$. Note that the corrections to $H_{\text {ref }}$ resulting from the system-environment interaction (i.e., the Lamb shift Hamiltonian) have been neglected in Eq. (7), and that the rotating wave approximation is implicit in its derivation. Therefore the time scale of the system $\tau_{S} \sim \max \left\{g^{-1}, \omega_{\alpha}^{-1}\right\}$ must be much smaller than the dissipation time, i.e., $\tau_{S} \ll \gamma^{-1}$.

Our dissipative system-environment coupling operators $\sqrt{\gamma} \sigma_{x_{\alpha}}$ give rise to six open decay channels (for each $\alpha$ ), associated with the frequencies $\left\{ \pm \omega_{\alpha}, \pm \omega_{\alpha} \pm g\right\}$. Consider, for instance, the Lindblad operators within the cold dissipator $\mathcal{D}_{c}$ : While the operators $A_{c, \pm \omega_{c}}$ produce transitions

$$
\left|0_{w} 0_{h} 0_{c}\right\rangle \leftrightarrow\left|0_{w} 0_{h} 1_{c}\right\rangle, \quad\left|1_{w} 1_{h} 0_{c}\right\rangle \leftrightarrow\left|1_{w} 1_{h} 1_{c}\right\rangle,
$$

in which the cold bath exchanges energy locally with the cold qubit only, the remaining operators, e.g., $A_{c, \pm \omega_{c}+g}$, are instead responsible for processes like

$$
\begin{aligned}
& \left|1_{w} 0_{h} 0_{c}\right\rangle \leftrightarrow\left(\left|1_{w} 0_{h} 1_{c}\right\rangle \pm\left|0_{w} 1_{h} 0_{c}\right\rangle\right) / \sqrt{2}, \\
& \left|0_{w} 1_{h} 1_{c}\right\rangle \leftrightarrow\left(\left|1_{w} 0_{h} 1_{c}\right\rangle \mp\left|0_{w} 1_{h} 0_{c}\right\rangle\right) / \sqrt{2},
\end{aligned}
$$

in which bath $c$ now exchanges energy with the work and hot qubit as well, in a delocalized way. It is in this sense that we refer to $\mathcal{D}_{\alpha}$ as modeling a delocalized dissipation effect.

Of course, as the limit of vanishing coupling $g$ is approached, the rates $\Gamma_{\alpha, \pm \omega+g}$ and $\Gamma_{\alpha, \pm \omega-g}$ become equal, and all delocalized transitions tend to compensate each other. For $g=0$, only two (local) decay channels remain open for each bath, namely $A_{\alpha, \pm \omega_{\alpha}}(0) \propto \sigma_{\alpha, \mp} \otimes \mathbb{1}_{\bar{\alpha}}$, which stands for the usual ladder operators for qubit $\alpha$ (the remaining qubits are denoted as $\bar{\alpha}$ ). The idealized model of Refs. [5-7] is recovered in this limit. Note that since the frequencies appear exponentiated in the spectral correlation tensor, delocalized dissipation effects are intuitively expected to be still relevant (i.e., $\Gamma_{ \pm \omega_{\alpha}+g} \not \nsucc \Gamma_{ \pm \omega_{\alpha}-g}$ ) even for arbitrarily small $g \lll 1$ (see Appendix A).

Equipped with the stationary solutions of the Markovian master equation (7), we can compute the central quantities of our study, namely, the rates at which energy from each bath is fed into the system, i.e., the heat currents. These are given as
$\dot{\mathcal{Q}}_{\alpha}=\operatorname{Tr}\left\{H_{\text {ref }} \mathcal{D}_{\alpha}\left[\varrho^{\infty}\right]\right\}$ [24]. In particular, we refer to $\dot{\mathcal{Q}}_{c}$ as the cooling power. Therefore, the COP reads $\varepsilon=\dot{\mathcal{Q}}_{c} / \dot{\mathcal{Q}}_{w}[2,6]$.

\section{B. Delocalized dissipation and irreversibility}

Let us now comment on our intuition linking delocalized dissipation with irreversibility in the operation of the machine. The Carnot COP $\varepsilon_{C}$ is realized at the upper limit of the cooling window Eq. (6), whenever $\varepsilon=\dot{\mathcal{Q}}_{c} / \dot{\mathcal{Q}}_{w}=\omega_{c} / \omega_{w}$ [6]. This would be the case (for arbitrary $g$ ) if one replaced the consistent Eq. (7) with a "localized" master equation such as

$$
\dot{\varrho}=-i\left[H_{\mathrm{ref}}, \varrho\right]+\sum_{\alpha} \mathscr{D}_{\alpha} \otimes \mathbb{1}_{\bar{\alpha}}[\varrho],
$$

like the one used in [5-7]. Here, the notation $\mathscr{D}_{\alpha} \otimes \mathbb{1}_{\bar{\alpha}}$ stands for some dissipator acting locally on qubit $\alpha$, in spite of using the full interacting Hamiltonian $H_{\text {ref }}=\sum_{\alpha} H_{0, \alpha}+H_{I}$ to account for the free dynamics.

Recall from the preceding considerations that such a localized model for the dissipation is only physically consistent in the limit of strictly vanishing $g$ [25]. On the contrary, if the realistic delocalized description of Eq. (7) is adopted, given any value of the qubit-qubit interaction, no matter how small, it becomes impossible to approach $\varepsilon_{C}$ arbitrarily closely, as illustrated in Fig. 2. There we plot the performance characteristics for the refrigerator according to Eq. (7) (solid red) and as results from the localized master equation of the type of Eq. (8) [5-7]. In both cases, the operation temperatures are $T_{h}=66.25$ and $T_{c}=4.78$. We also fix $T_{w}=127.33$, $\omega_{w}=56.87, g=0.1, \gamma=10^{-6}$, and $p_{i}=10^{-3}$. The only remaining free parameter $\omega_{c}$ is varied from 0 to the upper limit of the cooling window Eq. (6) $\omega_{c \text {, max }}$, and the cooling power $\dot{\mathcal{Q}}_{c}$ for each configuration is plotted versus the corresponding COP, normalized by the Carnot bound $\varepsilon_{C}$. The cooling powers are also normalized by their maximum values $\dot{\mathcal{Q}}_{c \text {, max }}$ : $8.39 \times 10^{-6}$ and $1.27 \times 10^{-6}$, respectively. Clearly, $\omega_{c}=0$ corresponds to $\dot{\mathcal{Q}}_{c}=\varepsilon=0$. In the ideal case of Eq. (8), $\omega_{c} \rightarrow \omega_{c, \text { max }}$ results in $\varepsilon \rightarrow \varepsilon_{C}$ and $\dot{\mathcal{Q}}_{c} \rightarrow 0$. However, any irreversibility would yield a COP not monotonically increasing

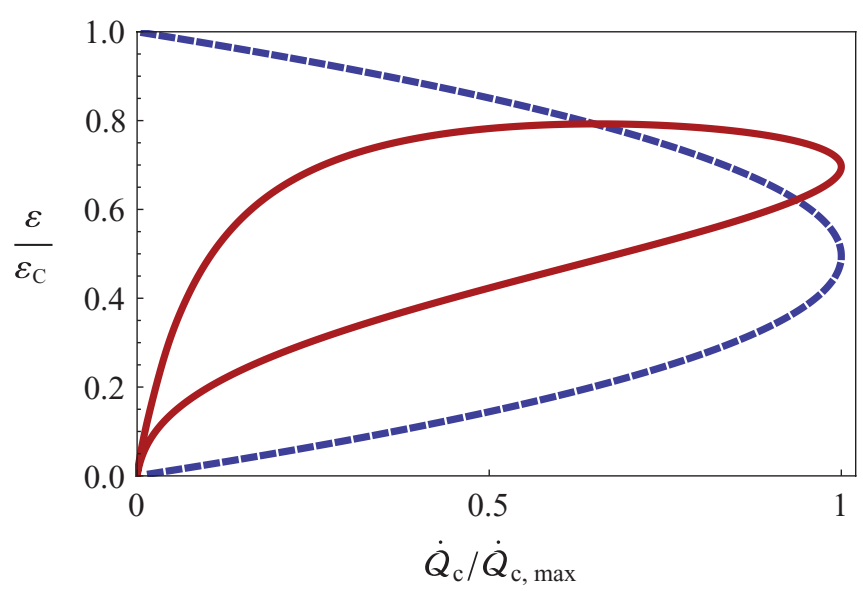

FIG. 2. (Color online) Comparison of the performance characteristics of the quantum absorption refrigerator according to the delocalized dissipative scheme of Eq. (7) (solid red) and to a "localized" master equation of the type Eq. (8) (dashed blue) for the same choice of parameters (see text for details). 
with $\omega_{c}$, and therefore a closed performance characteristic, as shown. This suggests that the unavoidable delocalization in the dissipative dynamics makes the refrigerator nonideal and somewhat wasteful, thus introducing a fundamental source of irreversibility that prevents it from cooling at the Carnot COP in practice.

\section{PERFORMANCE BOUNDS AT MAXIMUM POWER}

As we have just seen, in this three-qubit quantum absorption refrigerator, $\varepsilon_{C}$ cannot be approached arbitrarily closely in practice. It is therefore crucial to introduce an alternative tight bound on some performance indicator, such that its saturation would mark the functioning of the refrigerator as effectively optimal. A sensible figure of merit in this context is, for instance, the $\operatorname{COP} \varepsilon_{*}$ at maximum cooling power $\dot{\mathcal{Q}}_{c \text {, max }}$. One can then seek to devise a general upper bound for such a performance indicator, and to characterize a region within the space of the control parameters $\left\{\omega_{w}, T_{w}\right\}$ that allows for its saturation. This would provide useful work points for the efficient implementation of the machine.

To investigate this issue, we run extensive numerics on the stationary states of Eq. (7), globally optimizing $\varepsilon_{*}$ over all free parameters of the refrigerator, always under the consistency constraints implied by the Born-Markov and rotating wave approximations. We found that $\varepsilon_{*}$ is tightly upper bounded by

$$
\varepsilon_{*} \leqslant \frac{3}{4} \varepsilon_{C} .
$$

This is illustrated in Fig. 3, where $\varepsilon_{*} / \varepsilon_{C}$ was plotted for $10^{5}$ quantum absorption refrigerators whose free parameters were all sampled at random.

In order to get analytical insight into the role and possible origin of the performance limit, we resort to the much simpler mathematical description based on the localized master equation of Refs. [5-7]. Even though the irreversibility associated with delocalized dissipation is completely absent in this ideal case, meaning that the COP can reach $\varepsilon_{C}$ (albeit

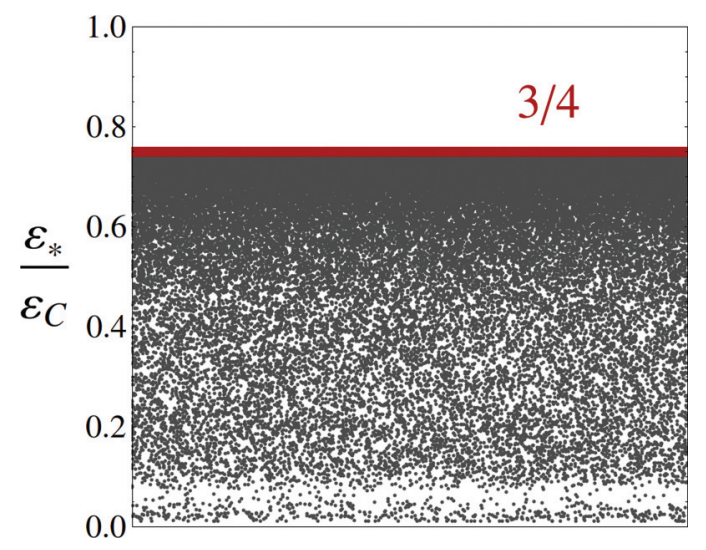

FIG. 3. (Color online) COP at maximum cooling power for $10^{5}$ random refrigerators, calculated from the stationary states of Eq. (7). The operation temperatures $T_{c}$ and $T_{h}$, as well as $T_{w}, \omega_{w}, g$, and $\gamma$, were chosen completely at random, always satisfying the constraints $k_{B} T_{\alpha} \gg \gamma$ (Born-Markov approximation) and $g \gg \gamma$ (rotating wave approximation). The value of $\omega_{c}$ yielding $\dot{\mathcal{Q}}_{c, \max }$ was found in each case (therefore fixing $\omega_{h}=\omega_{c}+\omega_{w}$ ) and the corresponding $\varepsilon_{*} / \varepsilon_{C}$ plotted. at vanishing power), the COP at maximum cooling power is, nonetheless, still tightly upper bounded when optimization over all parameters is carried out. Specifically, a similar numerical analysis shows that the bound turns out to be $\frac{1}{2} \varepsilon_{C}$ for the localized model. One can actually show this analytically, given $T_{w}$ and $\omega_{w}$ such that (i) $\omega_{w} / T_{w, h} \ll 1$ and (ii) $\omega_{c, \max } / T_{c} \ll 1$ (see Appendix $\mathrm{B}$ for a detailed proof), such a performance bound is saturated in the limit of large temperature difference $T_{c} / T_{h} \ll 1$.

Interestingly, coming back now to the realistic situation modeled by Eq. (7), with a consistent treatment of delocalized dissipation, one sees that conditions (i) and (ii) are also sufficient to saturate the $\frac{3}{4} \varepsilon_{C}$ performance bound on the COP at maximum power when working at large difference between the operating temperatures. These conditions, therefore, provide the desired design prescriptions for the practical implementation of efficient quantum absorption refrigerators of this kind. However, it is in order to remark that those are just sufficient conditions for optimal performance, and do not need to be necessarily met in order to attain a nearly optimal COP at maximum power: for instance, even the machine in Fig. 2 cools very close to the bound, despite having $\omega_{w} / T_{h} \sim 1$.

Finally, note that the fact that the performance bound differs quantitatively when the oversimplified localized picture is used instead ( $\frac{1}{2}$ vs $\frac{3}{4}$ as a fraction of $\varepsilon_{C}$ ) should not be surprising, as the underlying dissipative dynamics also encloses essential differences. The important points, however, are that the bound is also tight in that case, and that the analytical expression of the idealized stationary state is tractable enough and even proves insightful to obtain prescriptions for the saturation of the $\frac{3}{4} \varepsilon_{C}$ bound in the realistic model of Eq. (7).

\section{STATIONARY QUANTUM CORRELATIONS}

We finally investigate the stationary bipartite quantum correlations established in the refrigerator, focusing on the realistic dissipative model of Eq. (7). Given the structure of $\varrho^{\infty}$, the reduced states within the $2 \times 2$ bipartitions $w-h, w-c$, and $h-c$ are diagonal and therefore, unentangled and completely classical. We then consider the only two-dimensional subspaces of $\bigotimes_{\alpha} \mathcal{H}_{S, \alpha}$ which are in direct interaction according to $H_{\text {ref }}$, namely, those corresponding to the machine virtual qubit and the cold qubit. While no entanglement is found, more general quantum correlations measured by quantum discord [22,23,26] are always present in this relevant bipartition [27]. The structure of stationary quantum discord, analyzed in detail in the following, does not however exhibit any specific relationship either with the maximization of $\dot{\mathcal{Q}}_{c}$ or $\varepsilon$, or with the behavior of $\varepsilon_{*}$ or $\dot{\mathcal{Q}}_{c, \max }$, as the control parameters $\left\{\omega_{w}, T_{w}\right\}$ are varied. This supports the conclusion that the only essential quantum ingredient exploited by the machine is in fact the discreteness of its energy spectrum [5-7].

We recall that quantum discord is defined as

$$
D\left(\varrho_{A B}\right) \equiv \mathcal{I}\left(\varrho_{A B}\right)-\mathcal{I}\left(\sigma_{A B}\right),
$$

where the mutual information $\mathcal{I}\left(\varrho_{A B}\right)$ quantifies total correlations in the bipartite state $\varrho_{A B}$, and $\sigma_{A B}$ is the postmeasurement state after a minimally disturbing projective measurement on $B$. We refer the reader to Refs. [22,23,26] for details and interpretations of discord. 
The dissipative dynamics of Eq. (7) annihilates three-qubit $X$ states that have $\varrho_{36}^{\infty}=\varrho_{63}^{\infty}$ as the only nonzero matrix elements outside the main diagonal when expressed in the computational basis. If any of the qubits is traced out, the remaining $2 \times 2$ density matrix is diagonal and only involves the populations of $\varrho^{\infty}$. Therefore, entanglement and quantum discord in the bipartitions $w-h, w-c$, and $h-c$ vanish.

It is interesting to look instead at the only two-dimensional subspaces of $\bigotimes_{\alpha} \mathcal{H}_{S, \alpha}$ which are placed in direct interaction through $H_{I}$, that is, the machine virtual qubit and the cold qubit. The corresponding reduced state reads

$$
\begin{aligned}
\rho_{v c}^{\infty}= & \frac{P \varrho^{\infty} P}{\operatorname{tr} P \varrho^{\infty} P} \\
\text { with } P \equiv & \left|1_{w} 0_{h}\right\rangle\left\langle 1_{w} 0_{h}|+| 0_{w} 1_{h}\right\rangle\left\langle 0_{w} 1_{h}\right| \\
& +\left|0_{c}\right\rangle\left\langle 0_{c}|+| 1_{c}\right\rangle\left\langle 1_{c}\right| .
\end{aligned}
$$

When expressed in the basis $\left\{\left|1_{w} 0_{h}\right\rangle,\left|0_{w} 1_{h}\right\rangle\right\} \otimes\left\{\left|0_{c}\right\rangle,\left|1_{c}\right\rangle\right\}$, the two-qubit $X$ state $\rho_{v c}^{\infty}$ is given by

$$
\varrho_{v c}^{\infty}=\frac{1}{\mathcal{N}}\left(\begin{array}{cccc}
\varrho_{55}^{\infty} & 0 & 0 & 0 \\
0 & \varrho_{66}^{\infty} & \varrho_{36}^{\infty} & 0 \\
0 & \varrho_{36}^{\infty} & \varrho_{33}^{\infty} & 0 \\
0 & 0 & 0 & \varrho_{44}^{\infty}
\end{array}\right),
$$

where $\mathcal{N}$ is the normalization factor. According to the positivity-of-the-partial-transpose separability criterion $[28,29]$, the state $\varrho_{v c}^{\infty}$ is entangled if and only if

$$
\varrho_{36}^{\infty}>\frac{1}{2} \frac{\varrho_{44}^{\infty}+\varrho_{55}^{\infty}}{\varrho_{44}^{\infty}-\varrho_{55}^{\infty}}
$$

However, our stationary states are such that $\varrho_{36}^{\infty} \ll \varrho_{j j}^{\infty}$ for $j \in\{1, \ldots, 8\}$, and therefore no bipartite qubit entanglement can be present in them. On the contrary, one always finds nonzero stationary quantum discord between the machine virtual qubit and the cold qubit. In the search for the least disturbing local measurements for its quantification, we will restrict ourselves to projective measurements only. Since $\varrho_{v c}^{\infty}$ is an $X$ state, its discord can be computed analytically, using the formulas of Ref. [30].

In the first place, we fixed $T_{\alpha}$ and $\omega_{w}$ and looked at the stationary total $\left[\mathcal{I}\left(\varrho_{v c}\right)\right]$, quantum $\left[D\left(\varrho_{v c}\right)\right]$, and classical $\left[\mathcal{I}\left(\sigma_{v c}\right)\right]$ correlations between the machine virtual qubit and the cold qubit, to see whether they play any role in the maximization of the cooling power $\dot{\mathcal{Q}}_{c}$ for $0<\omega_{c} \leqslant \omega_{c \text {, max }}$ (see Fig. 4). Similarly to $\mathcal{Q}_{c}$, for intermediate values of $\omega_{w}$ all correlations are peaked around a certain value of $\omega_{c}$ which nevertheless usually differs from the frequency $\omega_{c, *}$ that maximizes $\dot{\mathcal{Q}}_{c}$ [cf. Figs. 4(c) and 4(d)]. Smaller work frequencies yield a monotonic behavior of correlations instead, as shown in Fig. 4(b), while larger values of $\omega_{w}$ reveal a more intricate structure [see Fig. 4(f)].

In Figs. 4(b) and 4(d), the measurements that maximize $\mathcal{I}\left(\sigma_{v c}\right)$ consist in, e.g., projections onto $\left|+{ }_{c}\right\rangle \equiv$ $\left(\left|0_{c}\right\rangle+\left|1_{c}\right\rangle\right) / \sqrt{2}$ and $\left|-{ }_{c}\right\rangle \equiv\left(\left|0_{c}\right\rangle-\left|1_{c}\right\rangle\right) / \sqrt{2}$ for any $\omega_{c}$. However, in Fig. 4(f) projective measurements in the computational basis of the cold qubit become optimal in the interval $14 \lesssim \omega_{c} \lesssim 66$. These discontinuous changes in the optimal measurement schemes result in a nondifferentiable classical correlations and quantum discord. In all three cases, the COP
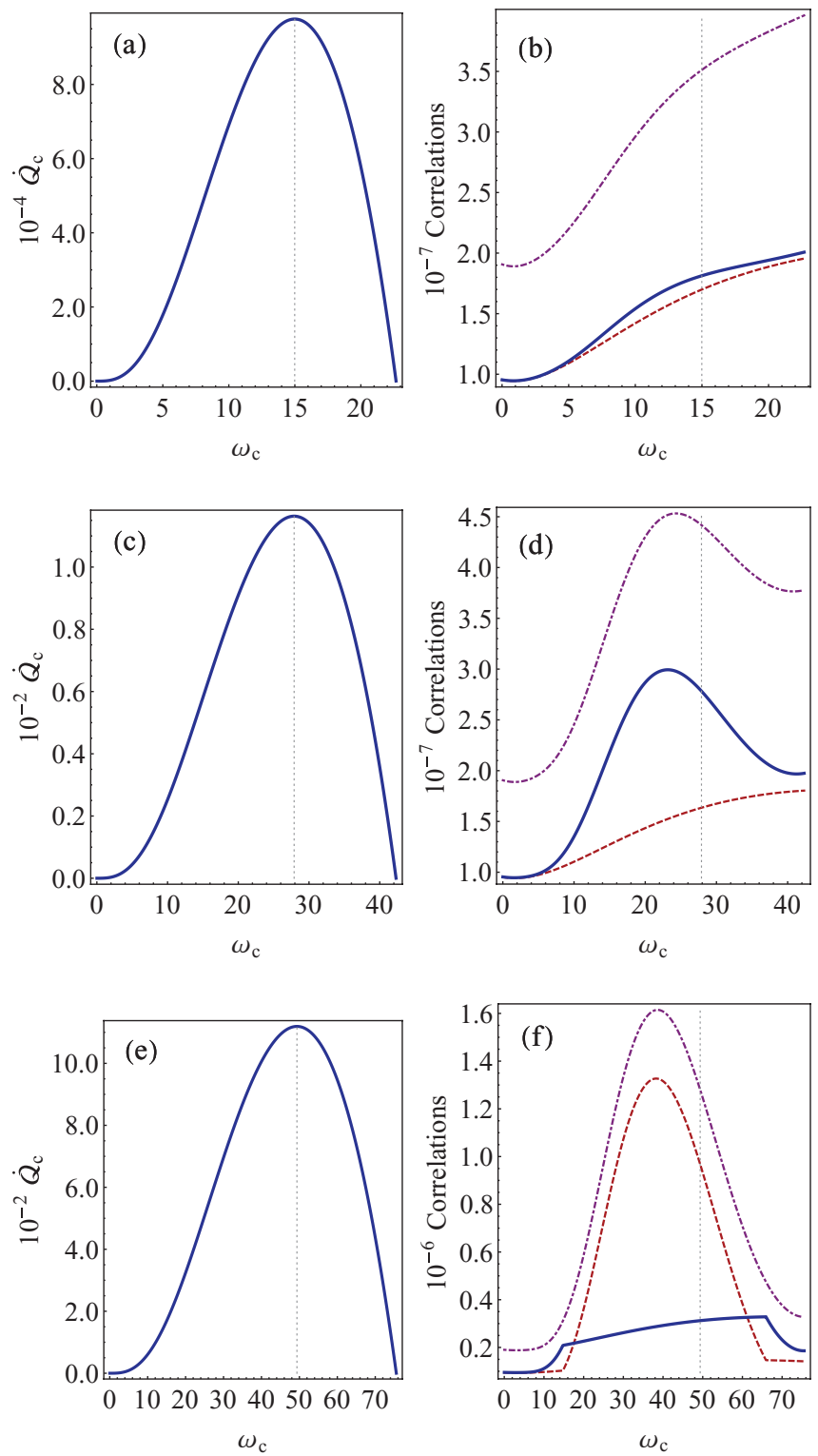

FIG. 4. (Color online) Left column: Cooling power $\dot{\mathcal{Q}}_{c}$ as a function of $\omega_{c}$ at fixed $T_{\alpha}, \omega_{w}, g$, and $\gamma$. Right column: Total (dot-dashed purple), classical (dashed red), and quantum (solid blue) correlations for $T_{w}=180, T_{h}=95, T_{c}=80, g=0.1, \gamma=10^{-6}$, and $\omega_{w}=10$ (a), (b), $\omega_{w}=15$ (b), (c), and $\omega_{w}=30$ (d), (e). The gray dotted line marks the position of the frequency $\omega_{c, *}$ maximizing $\dot{\mathcal{Q}}_{c}$.

increases linearly with $\omega_{c}$ and starts to decrease only as $\omega_{c, \max }$ is approached. It seems therefore clear that the maximization of $\mathcal{Q}_{c}$ and $\varepsilon$ are not related in any way to the only nonvanishing $2 \times 2$ stationary quantum correlations in the system.

We also ruled out any possible interplay between quantum discord at $\omega_{c, *}$ and the maximization of $\varepsilon_{*}$ and $\dot{\mathcal{Q}}_{c \text {, max }}$, using $\omega_{w}$ and $T_{w}$ as control parameters. In Fig. 5 we plot $\varepsilon_{*}$ and the corresponding quantum discord as a function of $\omega_{w}$ for different temperatures $T_{w}$. After increasing abruptly for small work frequencies, the COP at maximum power starts to decay as $\omega_{w}$ grows. When it comes to its temperature dependence, $\varepsilon_{*}$ seems to increase with $T_{w}$ as shown in Fig. 5. In Fig. 5(b), we see that for small $\omega_{w}$, quantum discord is also an increasing function of the work frequency. The optimal 

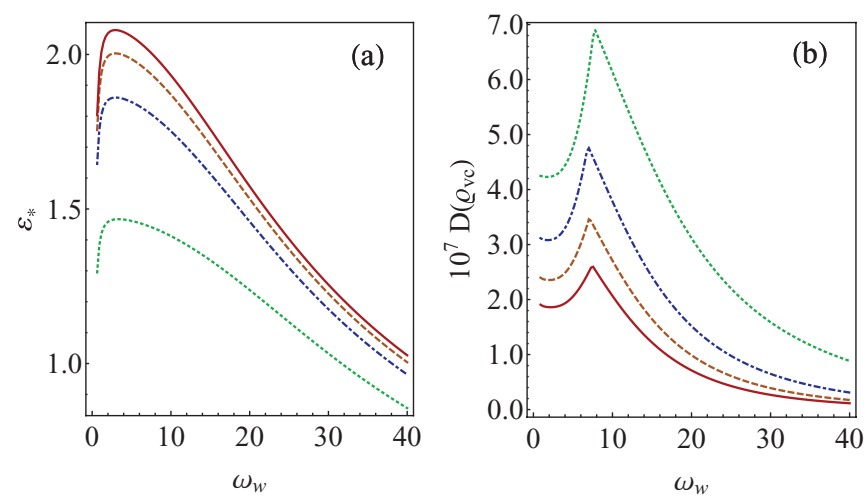

FIG. 5. (Color onlne) (a) COP at maximum cooling power $\varepsilon_{*}$ and (b) quantum discord at $\omega_{c, *}$ as functions of $\omega_{w}$ for different temperatures $T_{w}$. Parameters were chosen as $T_{h}=17, T_{c}=13, g=$ 0.1 , and $\gamma=2.5 \times 10^{-5}$, and $T_{w}=50$ (dotted green), $T_{w}=100$ (dotdashed blue), $T_{w}=150$ (dashed orange), and $T_{w}=200$ (solid red).

measurement scheme changes from $\left\{\left|+_{c}\right\rangle\left\langle+_{c}||-,_{c}\right\rangle\left\langle{ }_{c}\right|\right\}$ to $\left\{\left|0_{c}\right\rangle\left\langle 0_{c}|,| 1_{c}\right\rangle\left\langle 1_{c}\right|\right\}$ at some $\tilde{\omega}_{w}$, which produces a sharp maximum. For $\omega_{w}>\tilde{\omega}_{w}$, discord decays monotonically. At any fixed $\omega_{w}$, it is decreasing with the work temperature. The corresponding $\dot{\mathcal{Q}}_{c, \max }$ grows exponentially with $\omega_{w}$ and also increases as a function of $T_{w}$.

Even if the maximum discord at fixed $T_{w}$ does not coincide with the maximum of $\varepsilon_{*}$, it still marks a useful operation point of the refrigerator where a certain compromise between $\dot{\mathcal{Q}}_{c \text {, max }}$ and $\varepsilon_{*}$ is achieved. Our results also suggest that the COP at maximum power and the corresponding cooling power are enhanced, at fixed $\omega_{w}$ (and sufficiently small $g$ ), at the expense of the destruction of quantum correlations. It is possible, however, to increase these two figures of merit and yet build more quantumness in the system if one also leaves $\omega_{w}$ as a free parameter.

From all the preceding we see that there is no clear relationship between the quantumness of the bipartite qubitqubit correlations established in the stationary regime and the efficient performance of the refrigerator. In the idealized case of vanishing $g$, the ability of this quantum machine to saturate the Carnot COP comes from the discreteness of its energy spectrum, in contrast with any continuous "classical" counterpart [7]. Actually this discreteness and thermalization are the only essential building blocks for the basic operation of the refrigerator. It may not be surprising, then, that any residual quantumness of correlations appears in the system as a by-product rather than as fundamental resource for its enhanced performance.

\section{CONCLUSIONS}

We consistently studied the three-qubit quantum absorption chillers introduced in [5-7], adopting a physically meaningful system-bath interaction model. The resulting delocalized dissipation effects prevent the refrigerator a priori from cooling arbitrarily closely to the Carnot $\mathrm{COP} \varepsilon_{C}$, thus introducing unavoidable irreversibility in the stationary cooling process.

As an alternative to $\varepsilon_{C}$, a more useful performance bound had to be considered instead to assess the optimality of a given realization of such thermal devices. We chose to look at the
$\operatorname{COP} \varepsilon_{*}$ at maximum cooling power $\dot{\mathcal{Q}}_{c, \text { max }}$ and found that global optimization over all model parameters yields a tight upper bound on $\varepsilon_{*}$ of $\frac{3}{4} \varepsilon_{C}$. Sufficient conditions to saturate it in the limit of large temperature difference were also given.

The efficient performance of these machines was not found to relate in any obvious way to stationary bipartite total, classical, or quantum stationary correlations present in the system. Understanding the role played by the correlation properties of the environment in the performance limit could render the practical prescriptions for the realization of even more efficient quantum refrigerators accessible with presentday technology $[14,15]$ and will warrant further investigation. The extension of our results to a wider range of quantum absorption chillers will also be a subject of a future dedicated study.

\section{ACKNOWLEDGMENTS}

The authors are grateful to D. Girolami, N. Lo Gullo, K. Hovhannisyan, R. Kosloff, J. Goold, A. Acín, M. Perarnau, R. Vasile, R. Gallego, D. Cavalcanti, M. Navascués, and M. Huber for fruitful discussions and constructive criticism. This project was funded by the Spanish MICINN (Grant No. FIS2010-19998) and the European Union (FEDER), by the Canary Islands Government through the ACIISI fellowships (85\% cofunded by European Social Fund), and by the University of Nottingham through an Early Career Research and Knowledge Transfer Award and an EPSRC Research Development Fund Grant (No. PP-0313/36).

\section{APPENDIX A: DERIVATION OF THE MARKOVIAN MASTER EQUATION}

We will now consistently build the Markovian master equation Eq. (7) for the reduced state $\varrho$ of the three refrigerator qubits. The process starts by taking the interaction picture with respect to the free Hamiltonian $H_{F}=\sum_{\alpha} H_{0, \alpha}+H_{I}+$ $\sum_{\alpha} H_{B, \alpha}$. An initial preparation uncorrelated between system and environment is chosen so that $\rho(0)=\varrho(0) \otimes \chi$, where $\chi \equiv \bigotimes_{\alpha} \mathcal{Z}_{\alpha}^{-1} e^{-H_{B, \alpha} / T_{\alpha}}$. This choice guarantees on the one hand that the reduced evolution is a completely positive (and trace preserving) dynamical map [31] and on the other that the average of the bath operator $B_{\alpha} \equiv \sum_{\lambda} g_{\lambda}\left(a_{\alpha, \lambda}-a_{\alpha, \lambda}^{\dagger}\right)$ vanishes initially, $\operatorname{tr}_{B} B_{\alpha} \rho(0)=0$, and actually also at any later time as long as the Born approximation holds (see below).

The interaction picture Liouville-von Neumann equation for $\rho(t)$ is then suitably manipulated. Next, the Born or weak dissipation approximation, according to which $\rho(t) \simeq \varrho(t) \otimes$ $\chi$, is performed. The Markov approximation, which consists in neglecting any memory effects in the reduced evolution, finally leads to (see [24] for details)

$\dot{\tilde{\varrho}}=-\sum_{\alpha, \beta} \int_{0}^{\infty} d s \operatorname{tr}_{B}\left[H_{D, \alpha}(t),\left[H_{D, \beta}(t-s), \tilde{\varrho}(t) \otimes \chi\right]\right.$,

where $\tilde{\varrho}(t) \equiv e^{i H_{F} t} \varrho(t) e^{-i H_{F} t}$ stands for the interaction picture reduced state of the refrigerator qubits, and $H_{D, \alpha}(t) \equiv$ $e^{i H_{F} t} H_{D, \alpha} e^{-i H_{F} t}$. The dynamical map $\Phi(t, 0)$ evolving $\tilde{\varrho}(0)$ into $\tilde{\varrho}(t)$ that results from Eq. (A1) has the semigroup property under map composition: $\Phi(t, 0) \Phi(s, 0)=\Phi(t+s, 0)$, which 
implies that Eq. (A1) can be cast in the standard Lindblad form [32] of Eq. (7).

In order to achieve this, we shall decompose the system operators $\sigma_{x_{\alpha}}$ from the system-baths interaction term $H_{I}=$ $\sum_{\alpha} \sigma_{x_{\alpha}} \otimes B_{\alpha}$ into eigenoperators $\mathcal{A}_{\alpha, \omega}$ of $H_{\text {ref }}$ such that

$$
\sigma_{x_{\alpha}}=\sum_{\omega} \mathcal{A}_{\alpha, \omega}, \quad\left[H_{\mathrm{ref}}, \mathcal{A}_{\alpha, \omega}\right]=-\omega \mathcal{A}_{\alpha, \omega} .
$$

The non-Hermitian Lindblad or jump operators $\mathcal{A}_{\alpha, \omega}$ are defined as

$$
\mathcal{A}_{\alpha, \omega}=\sum_{\omega_{k}-\omega_{j}=\omega}^{\prime}|j\rangle\left\langle j\left|\sigma_{x_{\alpha}}\right| k\right\rangle\langle k|,
$$

where $|j\rangle$ is an eigenstate of $H_{\text {ref }}$ with energy $\omega_{j}$ and nondegeneracy is assumed. The eigenvalues of $H_{\mathrm{ref}}$ are $\left\{0, \omega_{w}, 2 \omega_{h}, \omega_{c}, \omega_{w}+\omega_{h}, \omega_{h}+\omega_{c}, \omega_{h}-g, \omega_{h}+g\right\}$ and their corresponding eigenvectors

$$
\begin{aligned}
& |1\rangle=\left|0_{w} 0_{h} 0_{c}\right\rangle, \quad|2\rangle=\left|1_{w} 0_{h} 0_{c}\right\rangle, \quad|3\rangle=\left|1_{w} 1_{h} 1_{c}\right\rangle, \\
& |4\rangle=\left|0_{w} 0_{h} 1_{c}\right\rangle, \quad|5\rangle=\left|1_{w} 1_{h} 0_{c}\right\rangle, \quad|6\rangle=\left|0_{w} 1_{h} 1_{c}\right\rangle, \\
& |7\rangle=\left(\left|1_{w} 0_{h} 1_{c}\right\rangle-\left|0_{w} 1_{h} 0_{c}\right\rangle\right) / \sqrt{2}, \\
& |8\rangle=\left(\left|1_{w} 0_{h} 1_{c}\right\rangle+\left|0_{w} 1_{h} 0_{c}\right\rangle\right) / \sqrt{2} .
\end{aligned}
$$

Therefore, from Eq. (A3) it is easy to see that there are only six open decay channels (i.e., transition frequencies $\omega=$ $\omega_{j}-\omega_{k}$ with nonzero $\mathcal{A}_{\alpha, \omega}$ ) for each bath $\alpha$, corresponding to $\left\{ \pm \omega_{\alpha}, \pm \omega_{\alpha} \pm g\right\}$. These jump operators are explicitly

$$
\begin{aligned}
\mathcal{A}_{w, \omega_{w}} & =\sqrt{\gamma}(|1\rangle\langle 2|+| 6\rangle\langle 3|), \\
\mathcal{A}_{w, \omega_{w}+g} & =\sqrt{\gamma}(|4\rangle\langle 8|-| 7\rangle\langle 5|) / \sqrt{2}, \\
\mathcal{A}_{w, \omega_{w}-g} & =\sqrt{\gamma}(|4\rangle\langle 7|+| 8\rangle\langle 5|) / \sqrt{2}, \\
\mathcal{A}_{h, \omega_{h}} & =\sqrt{\gamma}(|2\rangle\langle 5|+| 4\rangle\langle 6|), \\
\mathcal{A}_{h, \omega_{h}+g} & =\sqrt{\gamma}(|7\rangle\langle 3|+| 1\rangle\langle 8|) / \sqrt{2}, \\
\mathcal{A}_{h, \omega_{h}-g} & =\sqrt{\gamma}(|8\rangle\langle 3|-| 1\rangle\langle 7|) / \sqrt{2}, \\
\mathcal{A}_{c, \omega_{c}} & =\sqrt{\gamma}(|1\rangle\langle 4|+| 5\rangle\langle 3|), \\
\mathcal{A}_{c, \omega_{c}+g} & =\sqrt{\gamma}(|2\rangle\langle 8|-| 7\rangle\langle 6|) / \sqrt{2}, \\
\mathcal{A}_{c, \omega_{c}-g} & =\sqrt{\gamma}(|2\rangle\langle 7|+| 8\rangle\langle 6|) / \sqrt{2} .
\end{aligned}
$$

The remaining Lindblad operators are just given by the adjoint of these, since $\mathcal{A}_{\alpha, \omega}^{\dagger}=\mathcal{A}_{\alpha,-\omega}$.

The interaction picture system-environment coupling Hamiltonian may now be written as

$$
H_{I}(t)=e^{i H_{F} t} H_{I} e^{-i H_{F} t}=\sum_{\alpha, \omega} e^{-i \omega t} \mathcal{A}_{\alpha, \omega} \otimes B_{\alpha}(t),
$$

where $B_{\alpha}(t)=\sum_{\lambda} g_{\lambda}\left(a_{\lambda} e^{-i \omega_{\lambda} t}-a_{\lambda}^{\dagger} e^{i \omega_{\lambda} t}\right)$. Gathering all this back into Eq. (A1) yields

$$
\begin{aligned}
\dot{\tilde{\varrho}}= & \sum_{\alpha, \omega, \omega^{\prime}} e^{i\left(\omega^{\prime}-\omega\right) t} C_{\alpha, \omega}\left(\mathcal{A}_{\alpha, \omega} \tilde{\varrho} \mathcal{A}_{\alpha, \omega^{\prime}}^{\dagger}-\mathcal{A}_{\alpha, \omega^{\prime}}^{\dagger} \mathcal{A}_{\alpha, \omega} \tilde{\varrho}\right) \\
& + \text { H.c. },
\end{aligned}
$$

where the (complex) bath correlations $C_{\alpha, \omega}$ are defined as $C_{\alpha, \omega}=\int_{0}^{\infty} d s e^{i \omega s} \operatorname{tr}_{B} \chi B_{\alpha}(t) B_{\alpha}(t-s) \equiv \frac{1}{2} \Gamma_{\alpha, \omega}+i S_{\alpha, \omega}$.

Note that due to our choice of the initial preparation and provided that the Born approximation holds, all bath correlations depending on $\operatorname{tr}_{B} \chi B_{\alpha}(t) B_{\beta}(t-s)$ with $\alpha \neq \beta$ vanish, i.e., the baths are independent.

The details of $C_{\alpha, \omega}$ may also be worked out, exploiting the fact that the baths were prepared in a thermal equilibrium state [24]. Choosing $g_{\lambda} \propto \sqrt{\omega_{\lambda}}$, the spectral correlation tensor $\Gamma_{\alpha, \omega}$ reads

$$
\Gamma_{\alpha, \omega} \propto \omega^{3} e^{\beta \omega / 2}\left(\sinh \frac{\beta \omega}{2}\right)^{-1},
$$

where the proportionality constant is of order 1 and may be absorbed into $\gamma$.

Another important step towards the derivation of the master equation (7) is the assumption that the typical system time scales $\left|\omega-\omega^{\prime}\right|^{-1}$ with $\omega \neq \omega^{\prime}$ are much smaller than the relaxation time $\gamma^{-1}$, which allows one to discard all rapidly oscillating terms $\omega \neq \omega^{\prime}$ that average to zero in a coarse-grained picture of the reduced dynamics (rotating wave approximation). This leaves (we refer again to [24] for details)

$$
\dot{\tilde{\varrho}}=\sum_{\alpha, \omega} \Gamma_{\alpha, \omega}\left(\mathcal{A}_{\alpha, \omega} \tilde{\varrho} \mathcal{A}_{\alpha, \omega}^{\dagger}-\frac{1}{2}\left\{\mathcal{A}_{\alpha, \omega}^{\dagger} \mathcal{A}_{\alpha, \omega}, \tilde{\varrho}\right\}_{+}\right),
$$

where we have discarded the Lamb shift term $-i \sum_{\alpha, \omega} S_{\alpha, \omega}\left[\mathcal{A}_{\alpha, \omega}^{\dagger} \mathcal{A}_{\alpha, \omega}, \tilde{\varrho}\right]$ as is usually done when working in the quantum optical regime.

The only thing that remains to be done in order to recover Eq. (7), is to transform Eq. (A8) back into the Schrödinger picture by noting that

$\dot{\varrho}=-i H_{F} e^{-i H_{F} t} \tilde{\varrho} e^{i H_{F} t}+i e^{-i H_{F} t} \tilde{\varrho} e^{i H_{F} t} H_{F}+e^{-i H_{F} t} \dot{\tilde{\varrho}} e^{i H_{F} t}$.

This immediately yields Eq. (7) if one identifies $A_{\alpha, \omega}$ with $e^{-i H_{F} t} \mathcal{A}_{\alpha, \omega} e^{i H_{F} t}=e^{i \omega t} \mathcal{A}_{\alpha, \omega}$.

The reduced dynamics generated by Eq. (7) may be understood as a stochastic process in the Hilbert state space of the refrigerator qubits, in which the deterministic evolution of any pure state is interrupted by discontinuous quantum jumps $|\psi\rangle \mapsto \mathcal{N}^{-1} A_{\alpha, \omega}|\psi\rangle$, occurring at rates $\Gamma_{\alpha, \omega}$. The density matrix $\varrho(t)$ at any time $t$ is recovered as an ensemble average over stochastic trajectories [24,33].

It is clear that all interaction picture jump operators $\mathcal{A}_{\alpha, \omega}$ introduced above can produce delocalized dissipation (quantum jumps) in the sense discussed in the main article. As $g$ gets closer to zero, the rates $\Gamma_{\alpha, \pm \omega_{\alpha}+g}$ and $\Gamma_{\alpha, \pm \omega_{\alpha}-g}$ linearly approach each other, meaning that the jump processes $\mathcal{A}_{\alpha, \pm \omega+g}$ and $\mathcal{A}_{\alpha, \pm \omega-g}$ become equally likely, so that their delocalized contributions start to compensate. In the strict limit of $g=0$, only one transition frequency $\left(\omega_{\alpha}\right)$ is left for each bath, the corresponding Lindblad operator being just the sum $\mathcal{A}_{\alpha, \pm \omega}+\mathcal{A}_{\alpha, \pm \omega+g}+\mathcal{A}_{\alpha, \pm \omega-g} \propto \sigma_{\alpha, \mp}$ [cf. Eq. (A3)], which is a localized jump operator. Note that even if the difference $\left|\Gamma_{\alpha, \pm \omega+g}-\Gamma_{\alpha, \pm \omega-g}\right|$ depends linearly on $g \ll 1$, 
the exponentials in Eq. (A7) make it extremely sensitive to the qubit-qubit interaction strength, so that even a slight departure from the noninteracting case can make delocalized dissipation effects very important.

Let us finally comment on the underlying assumptions leading to Eq. (7). Even though the rotating wave approximation makes the problem much more tractable, it is not essential and one could just avoid it [cf. Eq. (A5)]. Situations in which the dissipation times become comparable to the system time scale (i.e., the realm of quantum Brownian motion) may then be accounted for, conceivably resulting in qualitative differences. It becomes important to account for the non-negligible renormalization effects of the systemenvironment interaction on the system itself in these cases. In contrast, within the quantum optical regime, the rotating wave approximation slightly modifies only the reduced dynamics but not the stationary states of the refrigerator, thus leaving our results unaffected.

If the baths are assumed to have some structure, the Markov approximation cannot be consistently performed. Nevertheless, as long as the dissipation strength remains sufficiently weak so that the Born approximation is still in place, no qualitative differences should be expected from what we report in the main article. Finally, if the dissipation becomes strong enough, the thermalization of a single isolated qubit in contact with its corresponding bath is no longer guaranteed, so that the basic operation of the refrigerator is compromised.

\section{APPENDIX B: ANALYTICAL DERIVATION OF THE PERFORMANCE BOUND $\frac{1}{2} \varepsilon_{C}$ UNDER LOCALIZED DISSIPATION}

Considering the localized dissipative model of [5-7], we shall now prove that its COP at maximum power $\varepsilon_{*}$ is upper bounded by $\frac{1}{2} \varepsilon_{C}$, whenever the conditions

$$
\begin{gathered}
\omega_{w} \ll T_{w, h}, \\
\omega_{w} \ll \tau \equiv \frac{T_{w}\left(T_{h}-T_{c}\right)}{T_{w}-T_{h}}
\end{gathered}
$$

are met, at large temperature difference $T_{c} / T_{h} \ll 1$.

Proof. Our starting point will be Eqs. (18) and (8)-(10) in Ref. [6], where the cooling power $\dot{\mathcal{Q}}_{c}$ was given as

$$
\dot{\mathcal{Q}}_{c, w}=q \frac{\Delta}{2+\frac{q^{2}}{2 g^{2}}+\sum_{\alpha} q_{\alpha}+\sum_{\alpha \beta} Q_{\alpha \beta} \Omega_{\alpha \beta}} \omega_{c, w}
$$

and

$$
\Delta=\frac{e^{-\left(\omega_{w}+\omega_{c}\right) / T_{h}}-e^{\omega_{w} / T_{w}} e^{-\omega_{c} / T_{c}}}{\left(1+e^{\omega_{w} / T_{w}}\right)\left(1+e^{\left(\omega_{w}+\omega_{c}\right) / T_{h}}\right)\left(1+e^{\omega_{c} / T_{c}}\right)},
$$

$\Omega_{\alpha \beta}= \begin{cases}\frac{1}{1+e^{-\omega_{\alpha} / T_{\alpha}}} \frac{e^{-\omega_{\beta} / T_{\beta}}}{1+e^{-\omega_{\beta} / T_{\beta}}}+\frac{e^{-\omega_{\alpha} / T_{\alpha}}}{1+e^{-\omega_{\alpha} / T_{\alpha}}} \frac{1}{1+e^{-\omega_{\beta} / T_{\beta}}} \quad(\alpha, \beta \neq h), \\ \frac{e^{-\omega_{\alpha} / T_{\alpha}}}{1+e^{-\omega_{\alpha} / T_{\alpha}}} \frac{e^{-\omega_{\beta} / T_{\beta}}}{1+e^{-\omega_{\beta} / T_{\beta}}}+\frac{1}{1+e^{-\omega_{\alpha} / T_{\alpha}}} \frac{1}{1+e^{-\omega_{\beta} / T_{\beta}}} \quad(\beta \neq \alpha=h), \\ \frac{1}{1+e^{-\omega_{\alpha} / T_{\alpha}}} \frac{1}{1+e^{-\omega_{\beta} / T_{\beta}}}+\frac{e^{-\omega_{\alpha} / T_{\alpha}}}{1+e^{-\omega_{\alpha} / T_{\alpha}}} \frac{1}{1+e^{-\omega_{\beta} / T_{\beta}}} \quad(\alpha \neq \beta=h), \\ \frac{e^{-\omega_{\alpha} / T_{\alpha}}}{1+e^{-\omega_{\alpha} / T_{\alpha}}} \frac{1}{1+e^{-\omega_{\beta} / T_{\beta}}}+\frac{1}{1+e^{-\omega_{\alpha} / T_{\alpha}}} \frac{1}{1+e^{-\omega_{\beta} / T_{\beta}}} \quad(\alpha=\beta=h) .\end{cases}$

Here, $q, q_{i}$, and $Q_{\alpha \beta}$ depend only on the three, possibly different, dissipation rates $p_{i}$, while $\Delta$ and $\Omega_{\alpha \beta}$ depend on all temperatures and frequencies. All we need to do is to find the $\omega_{c, *}$ that maximizes Eq. (B1) and then compute the corresponding $\varepsilon_{*}$.

First of all, note that conditions (i) and (ii) imply $\omega_{c} / T_{c} \ll 1$ for any $\omega_{c}<\omega_{c, \max }$, i.e., within the cooling window

$$
1>e^{-\omega_{c} / T_{c}}>e^{-\omega_{c, \max } / T_{c}}>e^{-\omega_{w} / \tau} \simeq 1-\frac{\omega_{w}}{T_{w}} .
$$

Due to the ordering $T_{w}>T_{h}>T_{c}$ in the bath's equilibrium temperatures, we must also have $\omega_{c} / T_{h} \ll 1$. Since $\omega_{h}=\omega_{w}+\omega_{c}$, this translates into $\omega_{h} / T_{h} \ll 1$. Therefore, conditions (i) and (ii) can be alternatively stated as

$$
\omega_{w, h, c} \ll T_{w, h, c} .
$$

Furthermore, in the limit of large temperature difference $T_{c} / T_{h} \ll 1$, assuming that $T_{c} / T_{h}$ is at least of order $\omega_{w} / T_{h}$ and $\omega_{w} / \tau$, we also have

$$
\frac{\omega_{c}}{T_{h}} \leqslant \frac{\omega_{c, \max }}{T_{h}}=\frac{\omega_{w}}{\tau} \frac{T_{c}}{T_{h}} \ll \frac{\omega_{w}}{T_{h}} .
$$

As a consequence, Eqs. (B2a) and (B2b) can be expressed as

$$
\begin{aligned}
\Delta & \simeq \frac{1}{8}\left(e^{-\omega_{w} / T_{h}}-e^{\omega_{w} / T_{w}} e^{-\omega_{c} / T_{c}}\right)+O\left(\frac{\omega_{\alpha}}{T_{\alpha}}\right), \\
\Omega_{\alpha \beta} & \simeq \frac{1}{2}+O\left(\frac{\omega_{\alpha}}{T_{\alpha}}\right) .
\end{aligned}
$$

In this regime, the denominator of $\dot{\mathcal{Q}}_{c}$ as given in Eq. (B1) becomes independent of $\omega_{c}$, so that its maximization is equivalent to that of Eq. (B6a). This yields

$$
\left(1-\frac{\omega_{c, *}}{T_{c}}\right) e^{\left(1-\omega_{c, *} / T_{c}\right)}=e^{\left(\omega_{w} / T_{w}-\omega_{w} / T_{h}\right)} .
$$

The solution to an equation of the form $x e^{x}=a$ may be expressed in terms of the Lambert $W$ function or product logarithm [34], as $x=W_{0}(a e)$. Therefore $\omega_{c, *}$ reads

$$
\omega_{c, *}=T_{c}\left[1-W_{0}\left(e^{\left(1+\omega_{w} / T_{w}-\omega_{w} / T_{h}\right)}\right)\right] .
$$

Among the properties of $W_{0}(z)$, we shall make use of its series expansion around $z=e$,

$$
W_{0}(z)=\frac{1}{2}+\frac{z}{2 e}+\cdots .
$$

Taking (i) into account again, $e^{\left(1+\omega_{w} / T_{w}-\omega_{w} / T_{h}\right)} \simeq 1+$ $\omega_{w} / T_{w}-\omega_{w} / T_{h}$, which, combined with Eqs. (B8) and (B9), results in

$$
\omega_{c, *} \simeq \frac{\omega_{w} T_{c}}{2}\left(\frac{1}{T_{h}}-\frac{1}{T_{w}}\right)
$$

The COP at maximum cooling power $\varepsilon_{*}=\omega_{c, *} / \omega_{w} \quad$ [6] normalized by $\varepsilon_{C}$ may be thus approximated by

$$
\frac{\varepsilon_{*}}{\varepsilon_{C}} \simeq \frac{T_{c}}{2 \varepsilon_{C}}\left(\frac{1}{T_{h}}-\frac{1}{T_{w}}\right)=\frac{1}{2}\left(1-\frac{T_{c}}{T_{h}}\right) \leqslant \frac{1}{2},
$$

which saturates in the limit of large temperature difference $T_{c} / T_{h} \ll 1$. 
[1] E. Geva and R. Kosloff, J. Chem. Phys. 104, 7681 (1996).

[2] J. P. Palao, R. Kosloff, and J. M. Gordon, Phys. Rev. E 64, 056130 (2001).

[3] A. Levy and R. Kosloff, Phys. Rev. Lett. 108, 070604 (2012).

[4] A. Levy, R. Alicki, and R. Kosloff, Phys. Rev. E 85, 061126 (2012).

[5] N. Linden, S. Popescu, and P. Skrzypczyk, Phys. Rev. Lett. 105, 130401 (2010).

[6] P. Skrzypczyk, N. Brunner, N. Linden, and S. Popescu, J. Phys. A 44, 492002 (2011).

[7] N. Brunner, N. Linden, S. Popescu, and P. Skrzypczyk, Phys. Rev. E 85, 051117 (2012).

[8] Y. Zhou and D. Segal, Phys. Rev. E 82, 011120 (2010).

[9] D. Gelbwaser-Klimovsky, R. Alicki, and G. Kurizki, Phys. Rev. E 87, 012140 (2013).

[10] S. Popescu, A. J. Short, and A. Winter, Nat. Phys. 2, 754 (2006),

[11] R. Dorner, J. Goold, C. Cormick, M. Paternostro, and V. Vedral, Phys. Rev. Lett. 109, 160601 (2012).

[12] D. Egloff, O. C. O. Dahlsten, R. Renner, and V. Vedral, arXiv:1207.0434.

[13] F. Giazotto, T. T. Heikkilä, A. Luukanen, A. M. Savin, and J. P. Pekola, Rev. Mod. Phys. 78, 217 (2006).

[14] Y.-X. Chen and S.-W. Li, Europhys. Lett. 97, 40003 (2012).

[15] D. Venturelli, R. Fazio, and V. Giovannetti, arXiv:1210.3649.

[16] O. Abah, J. Roßnagel, G. Jacob, S. Deffner, F. SchmidtKaler, K. Singer, and E. Lutz, Phys. Rev. Lett. 109, 203006 (2012).

[17] F. Curzon and B. Ahlborn, Am. J. Phys. 43, 22 (1975).
[18] S. Velasco, J. M. M. Roco, A. Medina, and A. C. Hernández, Phys. Rev. Lett. 78, 3241 (1997).

[19] M. Esposito, R. Kawai, K. Lindenberg, and C. Van den Broeck, Phys. Rev. Lett. 105, 150603 (2010).

[20] Y. Wang, M. Li, Z. C. Tu, A. C. Hernández, and J. M. M. Roco, Phys. Rev. E 86, 011127 (2012).

[21] A. E. Allahverdyan, K. Hovhannisyan, and G. Mahler, Phys. Rev. E 81, 051129 (2010).

[22] H. Ollivier and W. H. Zurek, Phys. Rev. Lett. 88, 017901 (2001).

[23] L. Henderson and V. Vedral, J. Phys. A 34, 6899 (2001).

[24] H. Breuer and F. Petruccione, The Theory of Open Quantum Systems (Oxford University Press, New York, 2002).

[25] N. Lo Gullo (private communication).

[26] K. Modi, A. Brodutch, H. Cable, T. Paterek, and V. Vedral, Rev. Mod. Phys. 84, 1655 (2012).

[27] A. Ferraro, L. Aolita, D. Cavalcanti, F. M. Cucchietti, and A. Acín, Phys. Rev. A 81, 052318 (2010).

[28] A. Peres, Phys. Rev. Lett. 77, 1413 (1996).

[29] M. Horodecki, P. Horodecki, and R. Horodecki, Phys. Lett. A 223, 1 (1996)

[30] M. Ali, A. R. P. Rau, and G. Alber, Phys. Rev. A 81, 042105 (2010).

[31] A. Shabani and D. A. Lidar, Phys. Rev. Lett. 102, 100402 (2009).

[32] G. Lindblad, Commun. Math. Phys. 48, 119 (1976).

[33] K. Molmer, Y. Castin, and J. Dalibard, J. Opt. Soc. Am. B 10, 524 (1993).

[34] R. M. Corless, G. H. Gonnet, D. E. G. Hare, D. J. Jeffrey, and D. E. Knuth, Adv. Comput. Math. 5, 329 (1996). 